\title{
Bilateral hypertrophic olivary degeneration after cerebral infarction
}

\author{
Franz A Fellner ${ }^{1,2 *}$ and Georg Hagleitner ${ }^{1}$ \\ ${ }^{1}$ Central Radiology Institute, Kepler University Hospital, Medical Faculty of the Johannes Kepler University, Linz, Austria \\ ${ }^{2}$ Medical Faculty of the Friedrich-Alexander-University of Erlangen-Nürnberg, Erlangen, Germany
}

\begin{abstract}
Hypertrophic olivary degeneration (HOD) is a rare finding secondary to focal lesions of the brain stem or the cerebellum. In such cases a functional system is involved - the Guillain-Mollaret triangle - composed of the dentate nucleus of the cerebellum of one side with the red nucleus and inferior olivary nucleus of the other side via the superior cerebellar peduncle and the central tegmental tract. Its hallmarks include hypertrophy of the inferior olivary nucleus with increased signal intensity on T2-weighted magnetic resonance images. It manifests with palatal tremor. Unilateral hypertrophic olivary degeneration is extremely rare, but more rare is the bilateral form as shown in this report of a 42-year old patient evolving after supra- and infratentorial infarction caused by basilar artery dissection.
\end{abstract}

\section{Introduction}

Hypertrophic olivary degeneration (HOD) was first reported by Oppenheim in the year of 1887 [1]. Hypertrophic olivary degeneration is a pathological phenomenon occuring after injury to the dentatorubro-olivary pathway. This anatomical triangle is formed by imaginary lines connecting the ipsilateral red and inferior olivary nuclei to the contralateral dentate nucleus of cerebellum via the central tegmental tract and the superior cerebellar peduncle. It is called the GuillainMollaret triangle, as it was described by Guillain and Mollaret in the year of 1931 [2].

Hypertrophic olivary degeneration represents the end result of a lesion that damages the neuronal connections within the GuillainMollaret triangle. Foix et al. in 1926 [3] used the term "transsynaptic degeneration" for this process, because it occurs after loss of synaptic input to a cell. It is considered to be a unique type of degeneration, because it is associated with enlargement, rather than atrophy of the affected structure. Pathologically, cell body enlargement, vacuolation of the cytoplasm, astrocytic hyperplasia and proliferation, demyelination and fibrillary gliosis are described [4].

Hypertrophic olivary degeneration can be seen on MR images as enlargement of the inferior olivary nucleus (ION) with increased signal on T2-weighted images. Increased olivary signal on T2-weighted images first appears 1 month after the inciting lesion and persists for at least 3 to 4 years. Olivary hypertrophy initially develops 4 to 6 months after the acute event and resolves by 3 to 4 years [5].

The inciting pathology of hypertrophic olivary degeneration includes infarction, hemorrhage, trauma (non-surgical and surgical) and tumor in a structure of the Guillain-Mollaret triangle. Hypertrophic olivary degeneration usually occurs unilaterally and ipsilateral to the lesion, if the lesion is in the brain stem. If the lesion is in the cerebellum, hypertrophic olivary degeneration occurs contralateral to the lesion. Bilateral olivary hypertrophy is indeed more rare than the unitlateral form of this entity. It can be explained by double innervations of each inferior olivary nucleus from either dentate nucleus or, if the primary lesion involves both, the central tegmental tract and the superior cerebellar peduncle [6]).

Myoclonic contractions of the soft palate, nystagmus and uncontrollable tremor, presumably caused by loss of inhibitory control, represent the effects of transsynaptic degeneration of the inferior olivary nuclei.

\section{Case report}

A 42-year-old man complained of neck pain for a few days. He tried to alleviate his pain by getting tai-chi massage. A few hours later he noted an abrupt onset of headache and his wife called the emergency because he fell into coma. Initial cranial CT was normal, no hemorrhage or infarction could be seen. Further tests revealed normal routine blood and CSF parameters.

On the initial MR examination multiple cerebellar ischemic lesions involving the dentate nucleus on both sides, in the area of the left posterior cerebral artery including the thalamic/hypothalamic area were seen (Figure 1).

On contrast enhanced MR angiography an irregular wall of the basilar artery, the left posterior cerebral artery and of the V4 segment of both vertebral arteries was found. Therefore, a dissection of the vertebral/basilar artery was assumed, despite the fact that even in catheter angiography no typical dissection membrane could be proved (Figure 2).

In the initial MR examination the medulla oblongata appeared completely normal in all sequences, even the T2-weighted sequences, (Figure 3).

${ }^{\star}$ Correspondence to: Franz A. Fellner, Central Radiology Institute, Kepler University Hospital, Medical Faculty of the Johannes Kepler University, Linz, Austria, E-mail: franz.fellner@kepleruniklinikum.at

Key words: bilateral hyertrophic olivary degeneration, cerebral infarction, arterial dissection, magnetic resonance (MR) imaging

Received: July 30, 2020; Accepted: August 17, 2020; Published: August 20, 2020 
On follow-up MR four months later, newly developed increased signal was found in the rostral portion of the medulla oblongata apart from the ischemic damage in the cerebellum bilaterally, occipital on the left side, in both thalamic/hypothalamic areas and in the mesencephalon, as known from the initial MR examination. The followup examination showed enlargement and increased signal intensity of both inferior olivary nuclei on T2-weighted images (Figure 4).

Enlargement and increased signal on T2-weighted images of inferior olivary nucleus in combination with ischemic lesions in the dentate nucleus of the cerebellum are findings that lead to the diagnosis of hypertrophic olivary degeneration. In our patient bilateral hypertrophic olivary degeneration was seen, because the ischemic lesions involved both dentate nuclei.

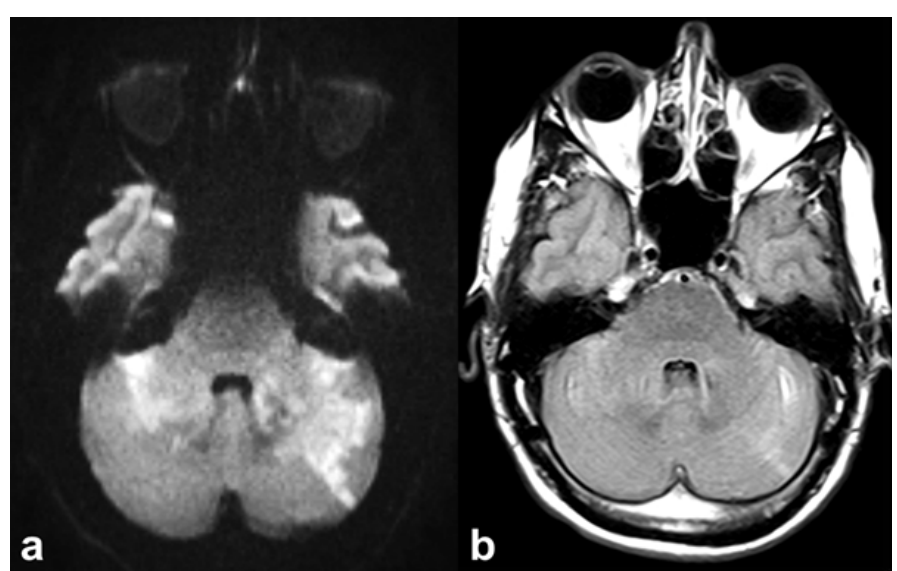

Figure 1. Initial MR examination shows bilateral infratentorial ischemic lesions in the acute stage. a) transverse diffusion-weighted image $\left(b=1000 \mathrm{~s} / \mathrm{mm}^{2}\right)$, b) transverse Fluid Attenuated Inversion Recovery (FLAIR) in transverse orientation

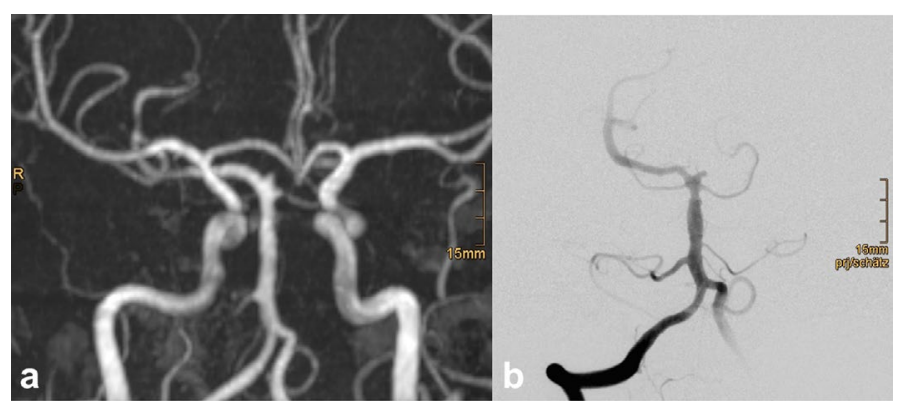

Figure 2. Contrast-enhanced MR-angiography of the initial MR examination (a) and subsequent catheter angiography (b) show irregularly outlined basilar artery, however, without dissection membrane

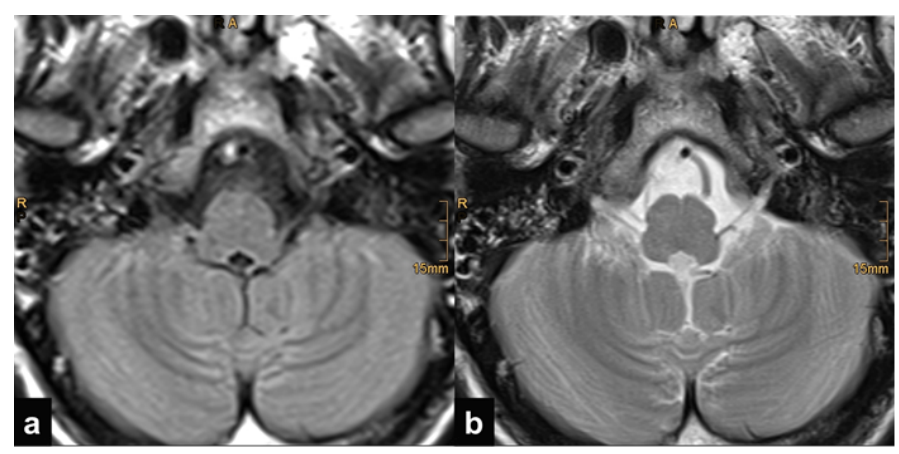

Figure 3. Initial MR examination: Normal visualization of the medulla oblongata containing the inferior olivary nuclei. a) FLAIR, b) T2-weighted turbo spin-echo (TSE) in transverse orientation

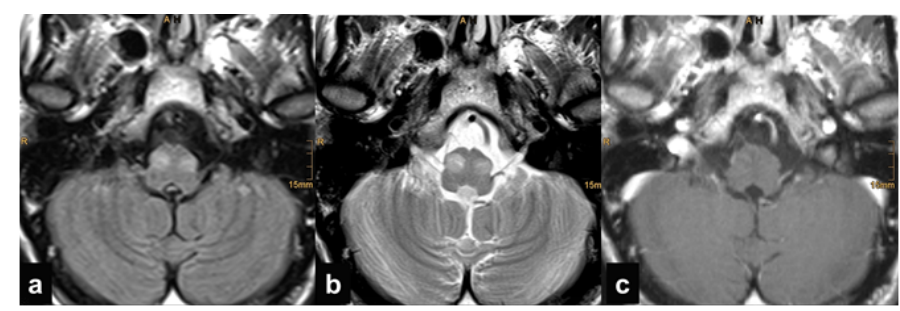

Figure 4. Follow-up MR four months later (transverse images). Bilaterally hypertrophic inferior olivary nuclei with increased signal on T2-weighted images without contrast enhancement on T1-weighted images after gadolinium injection. a) FLAIR, b) T2-weighted TSE, c) gadolinium enhanced T1-weighted spin-echo

Five months after the primary event the patient was referred to a specialized rehab hospital. In spite of intensive therapy the outcome of our patient was poor. Due to the extensive ischemic damage he developed a locked-in-syndrome.

\section{Discussion}

Considering the differential diagnosis of medullary lesions, increased signal on T2-weighted images within the pontomedullary region is not a specific imaging finding. It may be seen with a wide variety of pathologic processes including tumors, demyelinating lesions, infarction and inflammatory processes. But most neoplasms and infectious processes are expected to demonstrate significant enhancement. Therefore, a hyperintense lesion on T2-weighted images without gadolinium enhancement that is accompanied by enlargement of the inferior olivary nucleus, particularly if bilateral and symmetric, can be explained only by hypertrophic olivary degeneration [7]. If the oval shape of the olive becomes round with an apparent bulge, it must be classified as hypertrophic. The increased signal on T2-weighted images in hypertrophic olivary degeneration is strictly limited to one or both inferior olivary nucleus with sparing of the surrounding medullary tissue.

An important clue to the diagnosis hypertrophic olivary degeneration is the association of a lesion within the Guillain-Mollaret triangle. The presence of hypertrophic and in T2-weighted images hyperintense ION in association with another lesion in the GuillianMollaret triangle makes any diagnosis other than hypertrophic olivary degeneration highly unlikely. Only the knowledge of the components of the triangle of Guillain and Mollaret permits that the right diagnosis will be made. The delay in appearance of this olivary changes following lesions within the Guillain-Mollaret triangle further confirms the correct diagnosis.

\section{Disclosure}

No conflicts of interest. No grants or financial supports.

\section{References}

1. Oppenheim H (1887) Über Olivendegeneration bei Atheromatose der basalen Hirnarterien. Berl Klin Wochenschr 34: 638-639

2. Guillain G, Mollaret P (1931) Deux cas de myoclonies synchrones et rhythmes velopharyngo-laryngo-oculo-diaphragmatiques. Rev Neurol 2: 545-566.

3. Foix C (1926) Le syndrome myoclonique de la calotte. Rev Neurol 33: 942-956.

4. Duchen LW. General pathology of neurons and neuroglia. In: Hume Adams J; Duchen LW (1992). Greenfield's Neuropathology. 5. ed. New York:Oxford University Press, pp. 1-68.

5. Goyal M, Versnick E, Tuite P, Cyr JS, Kucharczyk W, et al. (2000) Hypertrophic olivary degeneration: metaanalysis of the temporal evolution of MR findings. Am J Neuroradiol 21: 1073-1077. [Crossref] 
6. Kitajima M, Korogi Y, Shimomura O, Sakamoto Y, Hirai T, et al. (1994) Hypertrophic olivary degeneration: MR imaging and pathologic findings. Radiology 192: 539-543. [Crossref]
7. Gerace C, Fele MR, Luna R, Piazza G (2006) Neurological picture. Bilateral hypertrophic olivary degeneration. J Neurol Neurosurg Psychiatry 77: 73.

Copyright: $\odot 2020$ Fellner FA. This is an open-access article distributed under the terms of the Creative Commons Attribution License, which permits unrestricted use, distribution, and reproduction in any medium, provided the original author and source are credited. 